Article

\title{
On the Generalized Kinetic Equation for Surface Gravity Waves, Blow-Up and Its Restraint
}

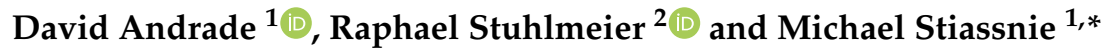 \\ 1 Faculty of Civil and Environmental Engineering, Technion-Israel Institute of Technology, Haifa 32000, Israel; \\ deandradep@technion.ac.il \\ 2 Centre for Mathematical Sciences, University of Plymouth, Plymouth PL4 8AA, UK; \\ raphael.stuhlmeier@plymouth.ac.uk \\ * Correspondence: miky@technion.ac.il
}

Received: 29 November 2018; Accepted: 24 December 2018; Published: 30 December 2018

\begin{abstract}
This article is concerned with the non-linear interaction of homogeneous random ocean surface waves. Under this umbrella, numerous kinetic equations have been derived to study the evolution of the spectral action density, each employing slightly different assumptions. Using analytical and numerical tools, and providing exact formulas, we demonstrate that the recently derived generalized kinetic equation exhibits blow up in finite time for certain degenerate quartets of waves. This is discussed in light of the assumptions made in the derivation, and this equation is contrasted with other kinetic equations for the spectral action density.
\end{abstract}

Keywords: ocean-waves; non-linear interactions; kinetic equation

\section{Introduction}

The dominant non-linear effect on the propagation of surface gravity waves in deep water is due to the resonant and almost resonant four-wave interactions discovered by Phillips [1]. This process of four-wave interaction is succinctly described by the Zakharov equation [2] which is a deterministic model accurate to third order in the wave steepness.

In the continuous case the Zakharov equation is written in terms of complex amplitudes $B(\mathbf{k}, t)$ as

$$
\frac{\partial B_{0}}{\partial t}=-i \iiint_{-\infty}^{\infty} T_{0,1,2,3} B_{1}^{*} B_{2} B_{3} \delta_{0+1-2-3} e^{i \Delta_{0,1}^{2,3} t} d \mathbf{k}_{1} d \mathbf{k}_{2} d \mathbf{k}_{3}
$$

Here, and elsewhere, subscripts denote a wavenumber component, so $B_{i}=B\left(\mathbf{k}_{i}, t\right)$. The wavenumber resonance condition for quartets of waves is evident in the delta function $\delta_{0+1-2-3}=\delta\left(\mathbf{k}_{0}+\mathbf{k}_{1}-\mathbf{k}_{2}-\mathbf{k}_{3}\right)$, while the frequency detuning $\Delta_{0,1}^{2,3}=\omega_{0}+\omega_{1}-\omega_{2}-\omega_{3}$ marks the departure from exact resonance. The asterisk denotes complex conjugation, while the kernel $T_{0,1,2,3}=T\left(\mathbf{k}_{0}, \mathbf{k}_{1}, \mathbf{k}_{2}, \mathbf{k}_{3}\right)$ is given in Krasitskii [3], and throughout this article the dispersion relation for deep water $\omega^{2}=g|\mathbf{k}|$, is used.

To describe averaged properties of a wave field, a statistical theory of wave evolution was developed in the 1950s. This culminated in Hasselmann's [4] derivation of what is often called the kinetic equation for water waves, or the Hasselmann equation. This equation describes the time evolution of the wave spectral density due to four-wave interactions in exact resonance.

The Hasselmann equation is derived under three basic premises that, in principle, pose limitations on its applicability. These assumptions are (i) spatial homogeneity and weak non-Gaussianity of the wave field, (ii) slow temporal evolution of the spectral density on a time-scale $\mathcal{O}\left(\varepsilon^{-4}\right)$, for $\varepsilon$ a typical wave steepness, and (iii) an assumption of quasi-stationarity for the fourth order cumulants. 
One of the consequences of assumption (iii) is that near resonant interactions are ruled out in Hasselmann's equation. In the deterministic description, near resonant interactions are responsible for the side-band instability of a uniform wave train. Stiassnie and Shemer [5] have further highlighted the equal importance of nearly resonant and exactly resonant interactions for the Zakharov equation. It is therefore desirable to include them in any statistical model.

The limitation imposed by quasi-stationarity was first studied in detail by Janssen [6], who derived a more general form of Hasselmann's equation. Janssen's equation essentially modifies (iii) by assuming the wave field to be strictly Gaussian at an arbitrary (but finite) initial time $t_{0}$. This has the immediate consequence that a Dirac delta function appearing in Hasselmann's equation is smeared out into a time dependent term involving the frequency detuning $\Delta_{0,1}^{2,3}$, thereby including near resonant interactions. Although Janssen's equation includes near-resonant interactions, which leads to a time evolution on the time scale $\mathcal{O}\left(\varepsilon^{-2}\right)$, it still uses a simplification that is justified under assumption (ii).

A simple physical example serves to demonstrate why it is advantageous to dispense with assumption (ii). Assuming a wave of period $T=10 \mathrm{~s}$ and steepness $\varepsilon=0.1$ leads to an evolution time-scale $t_{4}=T / \varepsilon^{4} \approx 27.7$ hours. To justify wave evolution under this paradigm, a sea-state must remain spatially homogeneous on such a time-scale, which is clearly an idealization. On the other hand, evolution on a faster time scale $t_{2}=T / \varepsilon^{2}$, or $16.6 \mathrm{~min}$ in the physical example above, would be a more suitable alternative.

A generalization of Janssen's approach was presented by Annenkov and Shrira [7]. Their result, called the generalized kinetic equation (GKE) is obtained as an intermediate step in the derivation of the kinetic equation, before assumptions (ii) and (iii) are imposed. This results in an equation valid on a fast time scale of order $\mathcal{O}\left(\varepsilon^{-2}\right)$. The equation was further generalized by Gramstad and Stiassnie [8], who also included higher order terms corresponding to the non-linear Stokes correction in their so-called phase averaged equation (PAE). Recently Gramstad and Babanin implemented the GKE, without Stokes' correction, in the third generation wave model WAVEWATCH-III [9].

The two equations dealt with in what follows will be Janssen's kinetic equation (henceforth JKE), and the generalized kinetic equation (GKE) of Annenkov and Shrira. While some recent efforts have been focused on the influence of inhomogeneities on the evolution of wave spectra, see Stuhlmeier and Stiassnie [10], thereby dispensing with assumption (i) along lines first suggested by Crawford et al. [11], it is important to put the homogeneous theory on sound footing. Thus, while JKE and GKE both essentially use the spatial homogeneity and weak non-Gaussianity of the wave field, they are differentiated by assumption (ii) on the slow evolution of the wave field. It will be shown that this seemingly small difference has a significant effect on the qualitative behavior of the solutions.

Our main result is that some solutions of the generalized kinetic equation exhibit blow-up. This means that the components of the spectrum tend to infinity in finite time. This raises a red flag on the GKE, as the blow-up is possible for initial conditions that are physically legitimate. Moreover, based only on the initial conditions we characterize when the blow-up occurs. One possible method to circumvent blow-up, by means of phase-mixing, is also explored in the appendix. On the other hand we show that solutions of the JKE always remain bounded, even in cases when there is blow-up in the GKE. Surprisingly, away from blow-up, periodic and bounded solutions of the GKE are qualitatively similar to those of the JKE.

\section{Theoretical Background}

Our starting point is the discrete version of Zakharov's equation, which assumes that the complex amplitudes can be written as

$$
B(\mathbf{k}, t)=\sum_{j=1}^{N} B_{j}(t) \delta\left(\mathbf{k}_{j}-\mathbf{k}\right)
$$


Substituting (2) into (1) yields the following system of ordinary differential equations for the discrete complex amplitudes $B_{j}$

$$
\frac{d}{d t} B_{j}=-i \sum_{m, n, p} T_{j, m, n, p} \delta_{j, m}^{n, p} e^{i \Delta_{j, m}^{n, p} t} B_{m}^{*} B_{n} B_{p}
$$

where $\delta_{j, m}^{n, p}$ denotes the Kronecker delta

$$
\delta_{j, m}^{n, p}= \begin{cases}1 & \text { when } \mathbf{k}_{j}+\mathbf{k}_{m}=\mathbf{k}_{n}+\mathbf{k}_{p} \\ 0 & \text { otherwise }\end{cases}
$$

Randomness is introduced by regarding the complex amplitudes as stochastic processes, where $\langle\cdot\rangle$ is used to denote an ensemble average. Assuming that $\left\langle B_{j}\right\rangle=0$ the next quantities of interest are the second-order moments, the pair correlators. Homogeneity, from assumption (i) above, imposes the following constraint on the correlators

$$
\left\langle B_{i}^{*}(t) B_{j}(t)\right\rangle=C_{j}(t) \delta_{i}^{j} .
$$

The set of functions $C_{j}(t)$ form the wave-action spectrum and $\delta_{i}^{j}$ is the Kronecker delta.

The evolution equation for the wave action is found by multiplying (3) by $B_{j}^{*}$ and adding its complex conjugate. The resulting equation is

$$
\frac{d}{d t} C_{j}=2 \sum_{m, n, p} T_{j, m, n, p} \delta_{j, m}^{n, p} \operatorname{Im}\left[e^{i \Delta_{j, m}^{n, p} t}\left\langle B_{j}^{*} B_{m}^{*} B_{n} B_{p}\right\rangle\right] .
$$

Higher order averages, such as the one appearing on the right hand side of (6), can be decomposed into products of lower order averages as follows

$$
\left\langle B_{j}^{*} B_{m}^{*} B_{n} B_{p}\right\rangle=C_{j} C_{m}\left(\delta_{j}^{n} \delta_{m}^{p}+\delta_{j}^{p} \delta_{m}^{n}\right)+\kappa_{j, m, n, p}
$$

where $\kappa$ is called the fourth-order cumulant. It is well known that all higher-order cumulants vanish for a Gaussian distribution, thus $\kappa$ indicates a deviation from Gaussianity.

Substituting (7) into (6), the evolution equation for the wave action becomes

$$
\frac{d}{d t} C_{j}=2 \sum_{m, n, p} T_{j, m, n, p} \delta_{j, m}^{n, p} \operatorname{Im}\left[e^{i \Delta_{j, m}^{n, p} t} \kappa_{j, m, n, p}\right]
$$

In order to find an equation for $\kappa$ we differentiate the fourth order average as follows

$$
\frac{d}{d t}\left\langle B_{j}^{*} B_{m}^{*} B_{n} B_{p}\right\rangle=\left\langle\frac{d B_{j}^{*}}{d t} B_{m}^{*} B_{n} B_{p}\right\rangle+\left\langle B_{j}^{*} \frac{d B_{m}^{*}}{d t} B_{n} B_{p}\right\rangle+\left\langle B_{j}^{*} B_{m}^{*} \frac{B_{n}}{d t} B_{p}\right\rangle+\left\langle B_{j}^{*} B_{m}^{*} B_{n} \frac{B_{p}}{d t}\right\rangle,
$$

and use (3) in the right hand side of (9) to yield

$$
\begin{aligned}
\frac{d}{d t}\left\langle B_{j}^{*} B_{m}^{*} B_{n} B_{p}\right\rangle & =i \sum_{u, v, w} T_{j, u, v, w} \delta_{j, u}^{v, w} e^{-i \Delta_{j, u}^{v, w} t}\left\langle B_{u} B_{v}^{*} B_{w}^{*} B_{m}^{*} B_{n} B_{p}\right\rangle \\
& +i \sum_{u, v, w} T_{m, u, v, w} \delta_{m, u}^{v, w} e^{-i \Delta_{m, u}^{v, w} t}\left\langle B_{j}^{*} B_{u} B_{v}^{*} B_{w}^{*} B_{n} B_{p}\right\rangle \\
& -i \sum_{u, v, w} T_{n, u, v, w} \delta_{n, u}^{v, w} e^{i \Delta_{n, u}^{v, w} t}\left\langle B_{j}^{*} B_{m}^{*} B_{u}^{*} B_{v} B_{w} B_{p}\right\rangle \\
& -i \sum_{u, v, w} T_{p, u, v, w} \delta_{p, u}^{v, w} e^{i \Delta_{p, u}^{v, w} t}\left\langle B_{j}^{*} B_{m}^{*} B_{n} B_{u}^{*} B_{v} B_{w}\right\rangle
\end{aligned}
$$


To close what would otherwise be an infinite hierarchy of equations we invoke the weak non-Gaussianity assumption. Akin to (7), one may decompose the sixth order averages in (10) into products of lower order averages and cumulants, as detailed in Lvov et al [12]. The terms are then ordered with respect to the small parameter $\varepsilon ; C_{j}=\mathcal{O}\left(\varepsilon^{2}\right)$ and $\kappa_{j, m, n, p}=o\left(\varepsilon^{4}\right)$, as in Gramstad and Stiassnie [8]. Therefore, up to order $\mathcal{O}\left(\varepsilon^{4}\right)$, the decomposition of the averages is

$$
\left\langle B_{j}^{*} B_{m}^{*} B_{n}^{*} B_{p} B_{u} B_{v}\right\rangle=C_{j} C_{m} C_{n}\left(\delta_{j}^{p}\left(\delta_{m}^{u} \delta_{n}^{v}+\delta_{m}^{v} \delta_{n}^{u}\right)+\delta_{j}^{u}\left(\delta_{m}^{v} \delta_{n}^{p}+\delta_{m}^{p} \delta_{n}^{v}\right)+\delta_{j}^{v}\left(\delta_{m}^{u} \delta_{n}^{p}+\delta_{m}^{p} \delta_{n}^{u}\right)\right) .
$$

Differentiating (7) with respect to $t$ and using Equation (10) together with (11) for the sixth-order terms we reach the following equation for $\kappa$, valid up to order $\mathcal{O}\left(\varepsilon^{4}\right)$

$$
\frac{d}{d t} \kappa_{j, m, n, p}=2 i T_{j, m, n, p} \delta_{j, m}^{n, p} e^{-i \Delta_{j, m}^{n, p} t}\left[C_{n} C_{p}\left(C_{j}+C_{m}\right)-C_{j} C_{m}\left(C_{n}+C_{p}\right)\right] .
$$

Equations (8) and (12) form the discrete version of the generalized kinetic equation first derived by Annenkov and Shrira [7] for a continuous wavenumber spectrum. Later, Gramstad and Stiassnie [8] derived their phase-averaged equation, by extending (11) to include terms up to $\mathcal{O}\left(\varepsilon^{6}\right)$. Our focus shall remain on the GKE, rather than the higher order extension.

The GKE contains both Janssen's and Hasselmann's kinetic equations. To derive Janssen's result we set $\kappa_{j, m, n, p}(0)=0$ and integrate Equation (12) from 0 to $t$, yielding

$$
\kappa_{j, m, n, p}=2 i T_{j, m, n, p} \int_{0}^{t} e^{-i \Delta_{j, m}^{n, p} s}\left[C_{n} C_{p}\left(C_{j}+C_{m}\right)-C_{j} C_{n}\left(C_{m}+C_{p}\right)\right] d s .
$$

Now we use assumption (ii). We assume that $\left[C_{n} C_{p}\left(C_{j}+C_{m}\right)-C_{j} C_{n}\left(C_{m}+C_{p}\right)\right]$ varies slowly in time and we extract it out of the integral to obtain

$$
\kappa_{j, m, n, p}=-2 T_{j, m, n, p}\left[C_{n} C_{p}\left(C_{j}+C_{m}\right)-C_{j} C_{n}\left(C_{m}+C_{p}\right)\right] \frac{\left(e^{-i \Delta_{j, m}^{n, p} t}-1\right)}{\Delta_{j, m}^{n, p}} .
$$

Substituting (14) into (8) yields a single equation for C:

$$
\frac{d}{d t} C_{j}=4 \sum_{m, n, p} T_{j, m, n, p}^{2} \delta_{j, m}^{n, p}\left[C_{n} C_{p}\left(C_{j}+C_{m}\right)-C_{j} C_{n}\left(C_{m}+C_{p}\right)\right] \frac{\sin \left(\Delta_{j, m}^{n, p} t\right)}{\Delta_{j, m}^{n, p}} .
$$

Equation (15) is the discrete version of Janssen's kinetic equation (denoted JKE), originally derived in continuous form by Janssen [6].

To elucidate the difference between the JKE (15) and the GKE (8) and (12), it is instructive to consider simple cases with few waves. The fundamental interaction in deep water is between quartets of waves, and the simplest such case consists of three distinct wavenumbers forming a degenerate quartet.

\section{Formulation for a Degenerate Quartet}

A degenerate quartet of waves consists of a discrete wave spectrum of the form

$$
B(\mathbf{k}, t)=B_{a}(t) \delta\left(\mathbf{k}_{a}-\mathbf{k}\right)+B_{b}(t) \delta\left(\mathbf{k}_{b}-\mathbf{k}\right)+B_{c}(t) \delta\left(\mathbf{k}_{c}-\mathbf{k}\right),
$$

where the wave-vectors $\mathbf{k}_{a}, \mathbf{k}_{b}$ and $\mathbf{k}_{c}$ satisfy the relation

$$
2 \mathbf{k}_{a}=\mathbf{k}_{b}+\mathbf{k}_{c} .
$$


The JKE and GKE thus describe the time evolution of the wave action $C_{j}=\left\langle\left|B_{j}\right|^{2}\right\rangle$, for $j=a, b, c$. For the GKE (8) and (12), taking all sums over only the three indices $a, b$ and $c$ yields, with the Kronecker delta function (17), the system of coupled ordinary differential equations

$$
\begin{aligned}
\frac{d}{d t} C_{a} & =4 T_{a, a, b, c} \operatorname{Im}\left[e^{i \Delta_{a, a}^{b, c} t} \kappa_{a, a, b, c}\right], \\
\frac{d}{d t} C_{b} & =-2 T_{a, a, b, c} \operatorname{Im}\left[e^{i \Delta_{a, a}^{b, c} t} \kappa_{a, a, b, c}\right], \\
\frac{d}{d t} C_{c} & =-2 T_{a, a, b, c} \operatorname{Im}\left[e^{i \Delta_{a, a}^{b, c} t} \kappa_{a, a, b, c}\right], \\
\frac{d}{d t} \kappa_{a, a, b, c} & =2 i T_{a, a, b, c} e^{-i \Delta_{a, a}^{b, c} t}\left[2 C_{a} C_{b} C_{c}-C_{a}^{2}\left(C_{b}+C_{c}\right)\right] .
\end{aligned}
$$

Likewise, the JKE (15) reduces to the following system of equations for the discrete wave action spectrum (16)

$$
\begin{aligned}
& \frac{d}{d t} C_{a}=16 T_{a, a, b, c}^{2}\left[C_{a} C_{b} C_{c}-\frac{1}{2} C_{a}^{2}\left(C_{b}+C_{c}\right)\right] \frac{\sin \left(\Delta_{a, a}^{b, c} t\right)}{\Delta_{a, a}^{b, c}}, \\
& \frac{d}{d t} C_{b}=-8 T_{a, a, b, c}^{2}\left[C_{a} C_{b} C_{c}-\frac{1}{2} C_{a}^{2}\left(C_{b}+C_{c}\right)\right] \frac{\sin \left(\Delta_{a, c}^{b, c} t\right)}{\Delta_{a, c}^{b, c}}, \\
& \frac{d}{d t} C_{c}=-8 T_{a, a, b, c}^{2}\left[C_{a} C_{b} C_{c}-\frac{1}{2} C_{a}^{2}\left(C_{b}+C_{c}\right)\right] \frac{\sin \left(\Delta_{a, c}^{b, c} t\right)}{\Delta_{a, a}^{b, c}} .
\end{aligned}
$$

\section{Analytic Solution for the GKE}

In this section we study the initial value problem associated with the system of equations (18)-(21). We begin by defining an auxiliary real function $Z(t)$ as the solution to the initial value problem

$$
\frac{d Z}{d t}=\operatorname{Im}\left[e^{i \Delta t} \mathcal{K}\right]
$$

with $Z(0)=0$. All subsequent calculations are based on the degenerate quartet, so we will omit subscripts and denote $\Delta=\Delta_{a, a}^{b, c}, \kappa=\kappa_{a, a, b, c}$ and $T=T_{a, a, b, c}$ where there is no risk of confusion.

In terms of $Z$, Equation (18)-(20) simplify to

$$
\frac{d}{d t} C_{a}=-2 \frac{d}{d t} C_{b}=-2 \frac{d}{d t} C_{b}=4 T \frac{d}{d t} Z
$$

Denoting the initial values by $C_{a}(0)=\rho_{a}, C_{b}(0)=\rho_{b}$ and $C_{c}(0)=\rho_{c}$ and integrating in time we find an expression for $C_{j}$ in terms of $Z$ :

$$
\begin{aligned}
& C_{a}(t)=\rho_{a}+4 T Z, \\
& C_{b}(t)=\rho_{b}-2 T Z, \\
& C_{c}(t)=\rho_{c}-2 T Z .
\end{aligned}
$$

Our goal is to find an equation that determines $Z(t)$. To this end we define a further auxiliary real function

$$
W=\operatorname{Re}\left[e^{i \Delta t} \mathcal{K}\right]
$$

Differentiating in time and using (21) yields

$$
\frac{d}{d t} W=-\Delta \operatorname{Im}\left[e^{i \Delta t} \mathcal{K}\right]+\operatorname{Re}\left[4 i T\left[C_{a} C_{b} C_{c}-\frac{1}{2} C_{a}^{2}\left(C_{b}+C_{c}\right)\right]\right]=-\Delta \frac{d}{d t} Z,
$$


which becomes, after integration,

$$
W=\operatorname{Re}\left[\kappa_{0}\right]-\Delta Z,
$$

where $\kappa(0)=\kappa_{0}$. Taking the time derivative of (25) yields

$$
\frac{d^{2} Z}{d t^{2}}=\Delta W+4 T\left[C_{a} C_{b} C_{c}-\frac{1}{2} C_{a}^{2}\left(C_{b}+C_{c}\right)\right] .
$$

Now we use Equations (27)-(29) to express the right hand side of (33) in terms of Z. The resulting equation has the form

$$
\frac{d^{2} Z}{d t^{2}}=P_{3}(Z)=b_{0}+b_{1} Z+b_{2} Z^{2}+b_{3} Z^{3}
$$

where the coefficients of $P_{3}$ are:

$$
\begin{aligned}
& b_{0}=\Delta \operatorname{Re}\left[\kappa_{0}\right]+4 T\left[\rho_{a} \rho_{b} \rho_{c}-\frac{1}{2} \rho_{a}^{2}\left(\rho_{b}+\rho_{c}\right)\right], \\
& b_{1}=-\Delta^{2}+4 T^{2}\left[4 \rho_{b} \rho_{c}-6 \rho_{a} \rho_{b}-6 \rho_{a} \rho_{c}+2 \rho_{a}^{2}\right], \\
& b_{2}=4 T^{3}\left[20 \rho_{a}-16 \rho_{b}-16 \rho_{c}\right], \\
& b_{3}=192 T^{4} .
\end{aligned}
$$

We multiply both sides of (34) by $d Z / d t$ and integrate

$$
\left(\frac{d Z}{d t}\right)^{2}=2 \int_{0}^{t} P_{3}(Z) \frac{d}{d t} Z d t=2 \int_{0}^{Z} P_{3}(z) d z
$$

obtaining a non-linear first order differential equation for $Z$

$$
\left(\frac{d Z}{d t}\right)^{2}=P_{4}(Z)=c_{0}+c_{1} Z+c_{2} Z^{2}+c_{3} Z^{3}+c_{4} Z^{4}
$$

The coefficients of $P_{4}$ are:

$$
\begin{aligned}
c_{0} & =\left(\operatorname{Im}\left[\kappa_{0}\right]\right)^{2}, \\
c_{1} & =2 b_{0}, \\
c_{2} & =b_{1}, \\
c_{3} & =\frac{2}{3} b_{2}, \\
c_{4} & =\frac{1}{2} b_{3} .
\end{aligned}
$$

\subsection{Analytical Solution for $\mathrm{Z}$}

We assume that $\kappa_{0}$ is real, so that 0 is always a root of $P_{4}$ (see (41)) and we are able to factor $P_{4}$ as

$$
P_{4}(Z)=Z Q_{3}(Z)
$$

where $Q_{3}(Z)$ is a cubic polynomial of the form

$$
Q_{3}(Z)=c_{1}+c_{2} Z+c_{3} Z^{2}+c_{4} Z^{3}
$$

with coefficients given by (42)-(45) (note that the JKE is derived assuming $\kappa_{0}=0$ (see (13)), and for a consistent comparison this initial condition is used in all subsequent examples). 
It is known that the nature of the roots of a third order polynomial is determined by the sign of the following discriminant

$$
D=18 c_{4} c_{3} c_{2} c_{1}-4 c_{3}^{3} c_{1}+c_{3}^{2} c_{2}^{2}-4 c_{4} c_{2}^{3}-27 c_{4}^{2} c_{1}^{2}
$$

If $D>0$ then all non-zero roots of $P_{4}$ are real and distinct. If $D=0$ then $P_{4}$ has a multiple non-zero real root. If $D<0$ then $P_{4}$ has two real roots and two complex conjugate roots. We refer to [13] for a detailed exposition about the discriminant of a cubic polynomial, and formula (48). Knowing the roots of $P_{3}$ allows us to find exact formulas for the solution of (40).

\subsubsection{Case $D>0$}

In all cases of interest, when $D>0$, the roots of $P_{4}$ can be written from largest to smallest as $Z_{4}>Z_{3}=0>Z_{2}>Z_{1}$. Therefore the solution of (40) can be found by inverting the integral

$$
t=\int_{0}^{Z} \frac{d z}{\sqrt{P_{4}(z)}}=-\int_{Z}^{0} \frac{d z}{\sqrt{P_{4}(z)}}=\int_{Z_{2}}^{Z} \frac{d z}{\sqrt{P_{4}(z)}}-\int_{Z_{2}}^{0} \frac{d z}{\sqrt{P_{4}(z)}}
$$

by applying formulas (254.00) of Byrd and Friedman [14]. Let

$$
K^{2}=\frac{Z_{2}\left(Z_{4}-Z_{1}\right)}{Z_{1}\left(Z_{4}-Z_{2}\right)}
$$

be the modulus of the Jacobian elliptic functions sn, cn and dn. Also let

$$
\begin{aligned}
\gamma & =\frac{2}{\sqrt{-\left(Z_{4}-Z_{2}\right) Z_{1}}} \\
\sin (\phi) & =\sqrt{\frac{Z_{1}\left(Z-Z_{3}\right)}{Z_{3}\left(Z-Z_{1}\right)}} .
\end{aligned}
$$

Then

$$
\mathrm{sn}^{-1}(\sin (\phi), K)=\mathrm{sn}^{-1}(1, K)+c_{4}^{1 / 2} \frac{t}{\gamma}=u,
$$

where $\mathrm{sn}^{-1}$ is the inverse of $\mathrm{sn}$. In term of $u$ the inverse of (49) is

$$
Z=\frac{Z_{2} Z_{1}\left(\operatorname{sn}^{2}(u, K)-1\right)}{Z_{2} \operatorname{sn}^{2}(u, K)-Z_{1}}
$$

We point out that the denominator of (54) never vanishes because $Z_{1} / Z_{2}>1$ whereas $\left|\operatorname{sn}^{2}(u, K)\right| \leq 1$. Finally we compute the value of $\operatorname{sn}(u, K)$ by means of addition formulas for elliptic functions. The resulting expression is

$$
\operatorname{sn}(u, K)=\frac{\operatorname{cn}\left(c_{4}^{1 / 2} t / \gamma, K\right) \operatorname{dn}\left(c_{4}^{1 / 2} t / \gamma, K\right)}{1-K^{2} \operatorname{sn}^{2}\left(c_{4}^{1 / 2} t / \gamma, K\right)} .
$$

The solution of (40), given by (54), is a bounded and periodic function of time with period

$$
T=\frac{2 \gamma \mathrm{sn}^{-1}(1, K)}{c_{4}^{1 / 2}}=\frac{2 \gamma}{c_{4}^{1 / 2}} \mathcal{K}(K),
$$

where $\mathcal{K}$ is the complete elliptic integral of the first kind. The qualitative behavior of the solution is shown in the left panel of Figure 1. The function $Z(t)$ oscillates back and forth between the roots $Z_{2}$ and $Z_{3}$ of the polynomial, starting from the initial value $Z_{3}=0$. 

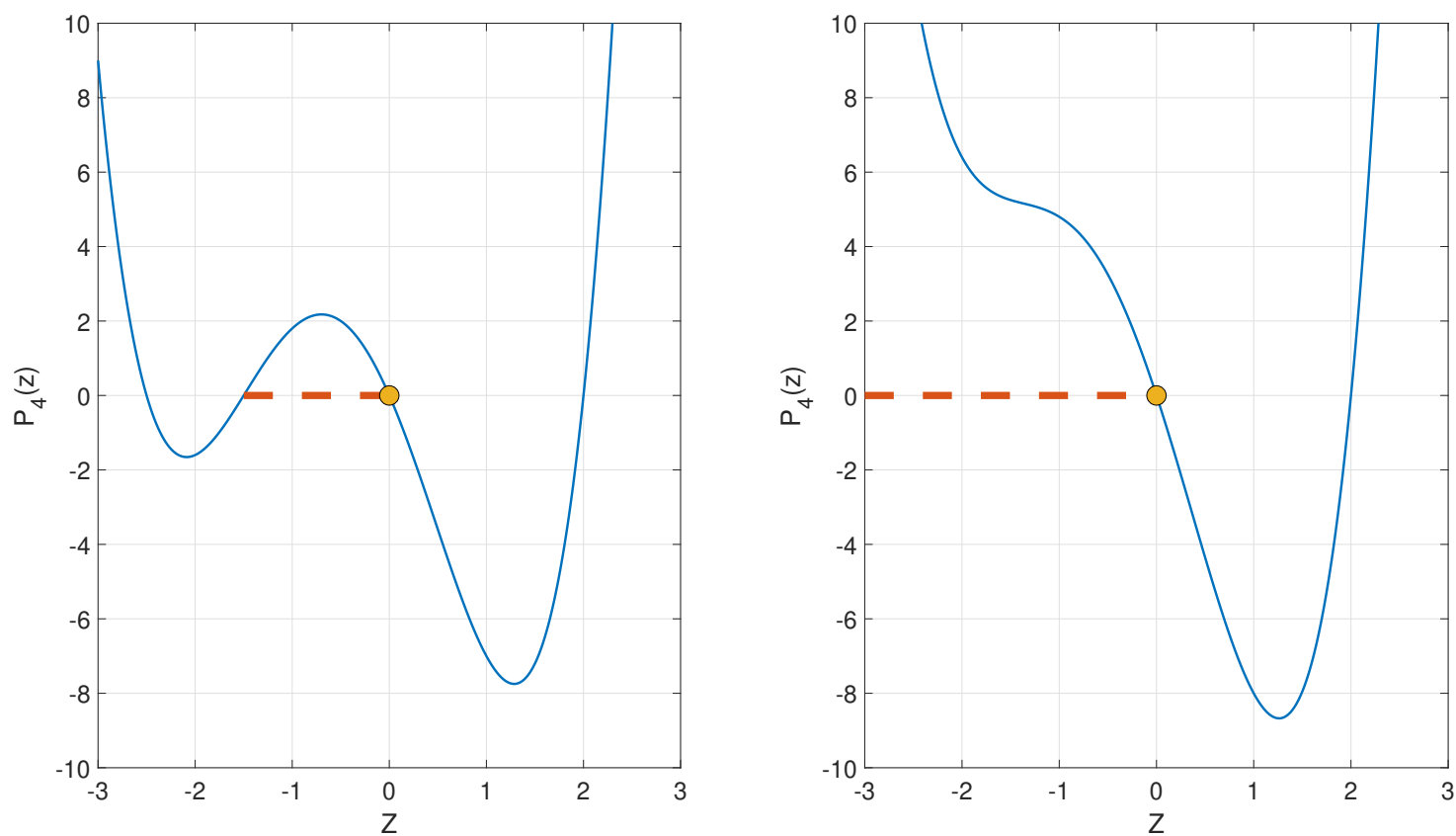

Figure 1. Schematic representation of the polynomial $P_{4}$. The red region (dashed line) represents the range of the function $Z(t)$. The yellow dot is the initial condition $Z(0)=0$. Left panel: $P_{4}$ has four real roots, $D>0$. Right panel: $P_{4}$ has two real roots and two complex roots, $D \leq 0$.

\subsubsection{Case $D \leq 0$}

In this case let $Z_{3}, Z_{3}^{*}, Z_{1}=0<Z_{2}$ be the roots of $P_{4}$. Notice that $D<0$ if and only if $\operatorname{Im}\left[Z_{3}\right] \neq 0$ and $D=0$ if and only if $\operatorname{Im}\left[Z_{3}\right]=0$. In either case the solution of (40) is found by inverting the integral

$$
t=\int_{0}^{Z} \frac{d z}{\sqrt{P_{4}(z)}}
$$

The value of (57) is found by applying formulas (260.00) of Byrd and Friedman [14]. Let

$$
K^{2}=\frac{\left(\left|Z_{3}\right|+\left|Z_{2}+Z_{3}\right|\right)^{2}-Z_{2}^{2}}{4\left|Z_{3}\right|\left|Z_{2}+Z_{3}\right|}
$$

be the elliptic modulus and define the parameters

$$
\begin{aligned}
\gamma & =\frac{1}{\sqrt{\left|Z_{3}\right|\left|Z_{2}+Z_{3}\right|}} \\
\cos (\phi) & =\frac{\left(\left|Z_{3}\right|-\left|Z_{2}+Z_{3}\right|\right) Z-Z_{2}\left|Z_{3}\right|}{\left(\left|Z_{3}\right|+\left|Z_{2}+Z_{3}\right|\right) Z-Z_{2}\left|Z_{3}\right|}
\end{aligned}
$$

Then

$$
\mathrm{cn}^{-1}(\cos (\phi), K)=-c_{4}^{1 / 2} \frac{t}{\gamma}=u .
$$

We invert this relation to find $Z$ as a function of $t$

$$
Z=\frac{Z_{2}\left|Z_{3}\right|(1-\operatorname{cn}(u, K))}{\left(\left|Z_{3}\right|-\left|Z_{2}+Z_{3}\right|\right)-\left(\left|Z_{3}\right|+\left|Z_{2}+Z_{3}\right|\right) \operatorname{cn}(u, K)} .
$$


In this case we expect the solution to blow up when the denominator of (62) vanishes. The critical time $T_{\infty}$ when this happens is

$$
T_{\infty}=\frac{\gamma}{c_{4}^{1 / 2}} \mathrm{cn}^{-1}\left(\frac{\left|Z_{3}\right|-\left|Z_{2}+Z_{3}\right|}{\left|Z_{3}\right|+\left|Z_{2}+Z_{3}\right|}, K\right)
$$

In the right panel of Figure 1 we show a schematic representation of the blow-up in terms of the roots of the quartic polynomial. The function $Z(t)$, initially at the root $Z_{1}=0$, tends towards $-\infty$ as $t$ goes from 0 to $T_{\infty}$.

\section{Analytic Solution of JKE}

Our analysis for the JKE follows the steps of the previous section. First we reduce the three coupled Equations (22)-(24) to a single equation for a new auxiliary function. To this end, let $z(t)$ be the solution of the initial value problem

$$
\frac{d z}{d t}=4 T\left[C_{a} C_{b} C_{c}-\frac{1}{2} C_{a}^{2}\left(C_{b}+C_{c}\right)\right] \frac{\sin (\Delta t)}{\Delta},
$$

with $z(0)=0$. The relationship between $z$ and $C$ is given by (27)-(29), as in Section 4 . This is substituted into the right-hand side of (64) to obtain the following equation for $z$

$$
\frac{d z}{d t}=P_{3}(z) \frac{\sin (\Delta t)}{\Delta}
$$

where $P_{3}(z)$ is a third order polynomial

$$
P_{3}(z)=d_{0}+d_{1} z+d_{2} z^{2}+d_{3} z^{3}
$$

with coefficients

$$
\begin{aligned}
& d_{0}=4 T\left[\rho_{a} \rho_{b} \rho_{c}-\frac{1}{2} \rho_{a}^{2}\left(\rho_{b}+\rho_{c}\right)\right] \\
& d_{1}=4 T^{2}\left[4 \rho_{b} \rho_{c}-6 \rho_{a} \rho_{b}-6 \rho_{a} \rho_{c}+2 \rho_{a}^{2}\right] \\
& d_{2}=4 T^{3}\left[20 \rho_{a}-16 \rho_{b}-16 \rho_{c}\right] \\
& d_{3}=192 T^{4} .
\end{aligned}
$$

Let $\lambda_{1}<\lambda_{2}<\lambda_{3}$ be the roots of $P_{3}$. Notice that $z(t)=\lambda_{i}$, for $i=1,2,3$, satisfies Equation (65). Moreover if $\lambda_{2}<0<\lambda_{3}$, then for all time $t, \lambda_{2}<z(t)<\lambda_{3}$. The subsequent analysis will be restricted to those cases encountered for physically relevant initial conditions-namely where $P_{3}$ has two negative roots and one positive root.

We integrate Equation (65) to obtain

$$
\left(\frac{z-\lambda_{1}}{\lambda_{1}}\right)^{\gamma_{1}}\left(\frac{z-\lambda_{2}}{\lambda_{2}}\right)^{\gamma_{2}}\left(\frac{\lambda_{3}-z}{\lambda_{3}}\right)^{\gamma_{3}}=\exp \left[\frac{d_{3}}{\Delta^{2}}(1-\cos (\Delta t))\right]
$$

with exponents

$$
\begin{aligned}
& \gamma_{1}=\frac{1}{\left(\lambda_{1}-\lambda_{2}\right)\left(\lambda_{1}-\lambda_{3}\right)}>0, \\
& \gamma_{2}=\frac{1}{\left(\lambda_{2}-\lambda_{1}\right)\left(\lambda_{2}-\lambda_{3}\right)}<0, \\
& \gamma_{3}=\frac{1}{\left(\lambda_{3}-\lambda_{1}\right)\left(\lambda_{3}-\lambda_{2}\right)}>0 .
\end{aligned}
$$


Equation (71) defines $z(t)$ implicitly. Nevertheless it shows that $z(t)$ has period $T=2 \pi / \Delta$. In case of exact resonance we take the limit as $\Delta \rightarrow 0$, obtaining

$$
\left(\frac{z-\lambda_{1}}{\lambda_{1}}\right)^{\gamma_{1}}\left(\frac{z-\lambda_{2}}{\lambda_{2}}\right)^{\gamma_{2}}\left(\frac{\lambda_{3}-z}{\lambda_{3}}\right)^{\gamma_{3}}=\exp \left[\frac{d_{3}}{2} t^{2}\right]
$$

This shows that in exact resonance conditions the solution is no longer periodic, but instead converges to the root $\lambda_{2}$.

In the examples presented below, rather than solving Equation (71) for $z(t)$ we integrate the system of Equations (22)-(24) directly using MATLAB's ode15s solver with absolute and relative tolerances set to $10^{-14}$.

\section{List of Examples}

In the following examples we consider three waves of the form $\mathbf{k}_{a}=k_{0}(1,0), \mathbf{k}_{b}=k_{0}(1+p, q)$ and $\mathbf{k}_{c}=k_{0}(1-p,-q)$ and, following Stuhlmeier and Stiassnie [10], we assume that the initial complex amplitudes are independent, Gaussian random variables, written as

$$
B_{j}(0)=\beta_{j} e^{i \phi_{j}},
$$

where the real amplitudes $\beta_{j}$ are Rayleigh distributed and the phases $\phi_{j}$ are uniformly distributed over the interval $[0,2 \pi)$.

Under these assumptions we have

$$
C_{j}(0)=\left\langle\left|B_{j}(0)\right|^{2}\right\rangle=\left\langle\beta_{j}^{2}\right\rangle=\frac{4}{\pi}\left\langle\beta_{j}\right\rangle^{2},
$$

and we relate the average value of $\beta_{j}$ to the wave amplitude by

$$
\left\langle\beta_{j}\right\rangle=\frac{\pi \varepsilon_{j}}{\left|\mathbf{k}_{j}\right|} \sqrt{\frac{\pi g}{2 \omega_{j}}} \text {, so that } C_{j}=2 g \pi^{2} \frac{\varepsilon_{j}^{2}}{\left|\mathbf{k}_{j}\right|^{2} \omega_{j}},
$$

where $\varepsilon_{j}$ are the wave slopes. Finally we set $\kappa_{a, a, b, c}(0)=0$, in order to have consistent initial conditions with which to compare GKE and JKE. In all calculations we take $k_{0}=1 \mathrm{~m}^{-1}, g=9.81 \mathrm{~m} \mathrm{~s}^{-2}$ and the period of the wave $\mathbf{k}_{a}$ is $T_{a}=2 \pi / \sqrt{g k_{0}} \approx 2.01 \mathrm{~s}$.

We shall consider four particular initial conditions below. In cases $\mathbf{a}$ and $\mathbf{b}$ we initially assume that the waves are collinear $(q=0)$ and fix the steepness $\varepsilon_{a}=0.15$ of the wave $\mathbf{k}_{a}$. Then we take $\epsilon=\varepsilon_{b}=\varepsilon_{c}$ to be the steepness of the waves $\mathbf{k}_{b}$ and $\mathbf{k}_{c}$. In cases $\mathbf{c}$ and $\mathbf{d}$ the wave slopes are fixed $\varepsilon_{a}=0.15, \varepsilon_{b}=0.05$ and $\varepsilon_{c}=0$ and we explore the nature of the solutions in the $(p, q)$-plane. Notice that $q \neq 0$ implies a two-dimensional disturbance. We recall that while solutions to the JKE are periodic, the behavior of the GKE is determined by the initial conditions via the discriminant $D$ given in (48). To visualize how wave slope and wavenumber impact the solutions of the GKE, the sign of $D$ is plotted in Figure 2. It establishes when the solutions remain bounded $(D>0)$ or tend to infinity in finite time $(D \leq 0)$. 

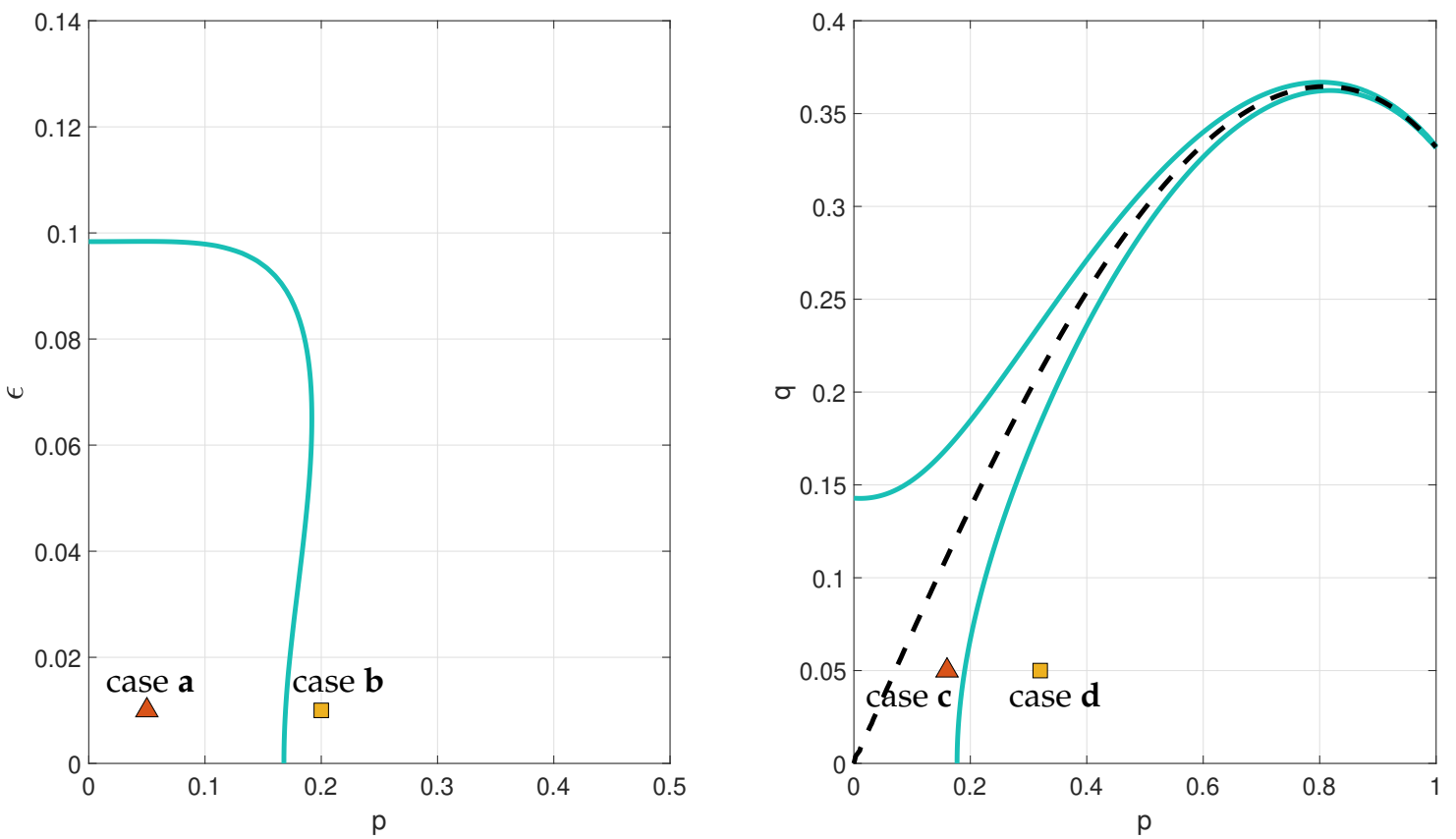

Figure 2. Plot of the discriminant (48) for varying initial conditions. The solid lines correspond to $D=0$, and the region they enclose about the origin is where $D<0$, and solutions of the GKE will diverge. The outer region is where $D>0$ and solutions of the GKE are periodic and bounded. Left panel: collinear waves, with varying $p$ and $\epsilon$. Case a has $(p=0.05, \epsilon=0.01)$ and case $\mathbf{b}$ has $(p=0.2, \epsilon=0.01)$. Right panel: fixed wave slopes, with varying $p$ and $q$. Case $\mathbf{c}$ has $(p=0.16, q=0.05)$ and case $\mathbf{d}$ has $(p=0.32, q=0.05)$. The dashed line shows the waves that are in exact resonance $2 \omega_{a}=\omega_{b}+\omega_{c}$.

The four cases a-d marked in Figure 2 are studied numerically, and the relevant parameters for these cases are summarized in Table 1.

Table 1. Degenerate quartets studied numerically for time-evolution.

\begin{tabular}{ccccccccc}
\hline & Wave Numbers & \multicolumn{3}{c}{ Steepness } & \multicolumn{2}{c}{ Nature of Solution } & Detuning \\
\hline case & $\boldsymbol{p}$ & $\boldsymbol{q}$ & $\boldsymbol{\varepsilon}_{\boldsymbol{a}}$ & $\boldsymbol{\varepsilon}_{\boldsymbol{b}}$ & $\boldsymbol{\varepsilon}_{\boldsymbol{c}}$ & GKE & JKE & $\boldsymbol{\Delta}_{\boldsymbol{a}, \boldsymbol{a}}^{\boldsymbol{b} \boldsymbol{c}\left(\mathbf{s}^{-\mathbf{1}}\right)}$ \\
\hline $\mathbf{a}$ & 0.05 & 0 & 0.15 & 0.01 & 0.01 & blow-up & periodic & 0.002 \\
$\mathbf{b}$ & 0.2 & 0 & 0.15 & 0.01 & 0.01 & periodic & periodic & 0.0317 \\
$\mathbf{c}$ & 0.16 & 0.05 & 0.15 & 0.05 & 0 & blow-up & periodic & 0.0161 \\
$\mathbf{d}$ & 0.32 & 0.05 & 0.15 & 0.05 & 0 & periodic & periodic & 0.0781 \\
\hline
\end{tabular}

\section{Comparison between GKE and JKE-Blow Up}

We compare the solutions of the GKE with those of the JKE for cases a and c (see Table 1), shown as triangles in Figure 2. Figure 3 shows the time evolution of case $\mathbf{a}$. In the upper panel we see the solution of the GKE that tends to infinity as time approaches $T_{\infty}=100.02 \mathrm{~s}$, see (63). The solution becomes physically meaningless as $C_{b}$ and $C_{c}$ become negative, prior to the critical time. On the other hand the solution of the JKE, on the lower panel of Figure 3 remains bounded, and is periodic with period $T=3207.19 \mathrm{~s}$. Adopting the notation $t_{n}=\left(2 \pi / \omega_{a}\right) / \varepsilon_{a}^{n}$ for the time scales we see that the blow-up occurs at $\mathcal{O}\left(t_{2}\right)$. The corresponding evolution for the JKE looks initially similar, but settles down to near equipartition of wave action, before eventually exhibiting periodicity on a time scale $\mathcal{O}\left(t_{4}\right)$.

The time evolution for case $\mathbf{c}$ is shown in Figure 4. The upper panel shows blow-up for the solution of the GKE. The critical time is $T_{\infty}=89.80 \mathrm{~s}$. This non-physical behavior is similar to case a (above) and occurs on the same time scale $\mathcal{O}\left(t_{2}\right)$. 


\section{Case a}
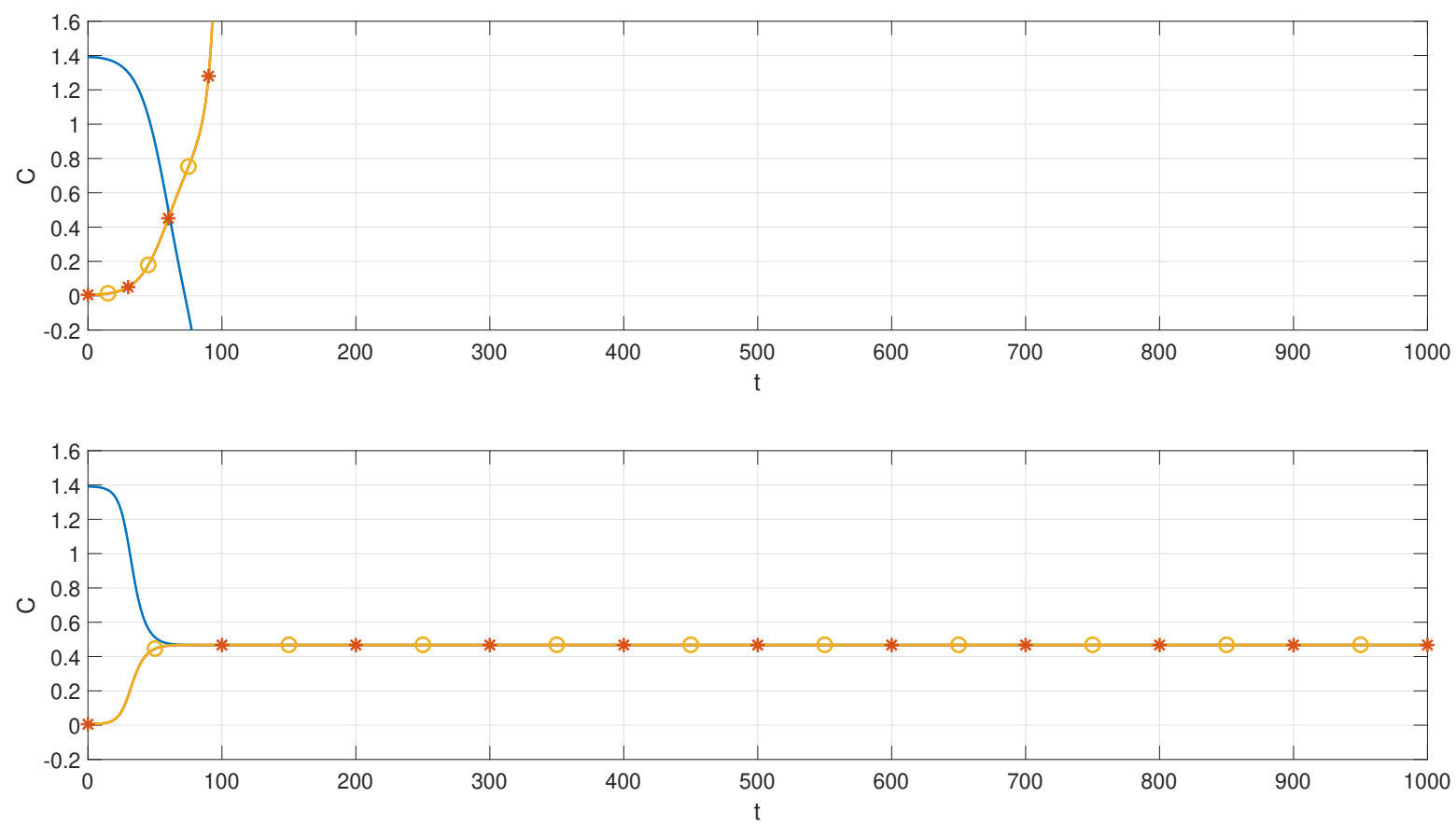

Figure 3. Time evolution of $C_{a}$ in blue (solid), $C_{b}$ in yellow (circles) and $C_{c}$ in red (asterisks) for $(p=0.05, \epsilon=0.01)$. Upper panel: Solution of the GKE. The blow-up time is $T_{\infty}=100.02 \mathrm{~s}$. Lower panel: Solution of the JKE. The period is $T=3207.19 \mathrm{~s}$. The time $t$ is in seconds.

\section{Case c}
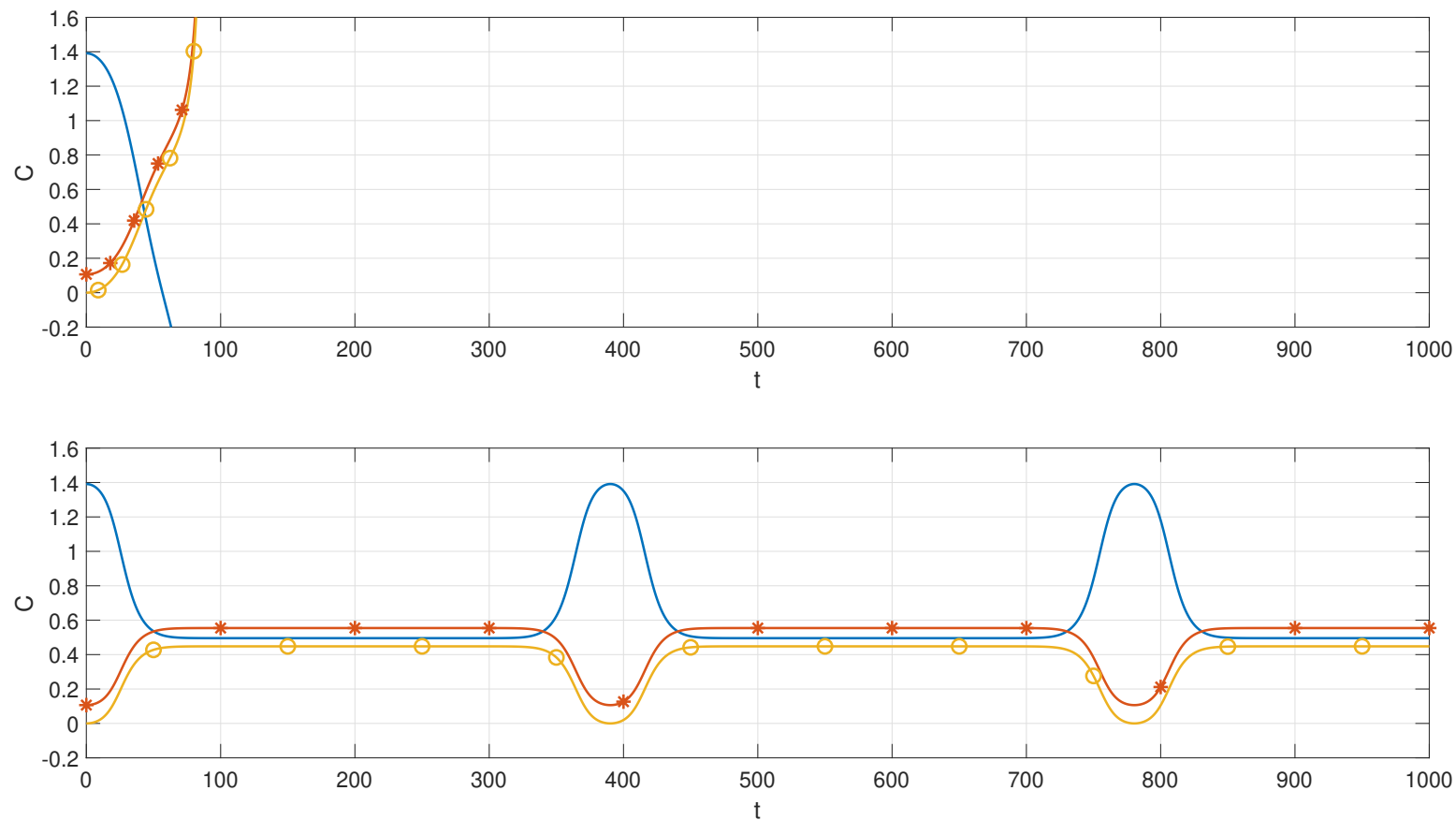

Figure 4. Time evolution of $C_{a}$ in blue, $C_{b}$ in yellow and $C_{c}$ in red for $(p=0.16, q=0.05)$. Upper panel: Solution of the GKE. The blow-up time is $T_{\infty}=89.80 \mathrm{~s}$. Lower panel: Solution of the JKE. The period is $T=390.19 \mathrm{~s}$. The time $t$ is in seconds.

On the lower panel we have the solution of the JKE. The system also reaches a state where the energy among the three wavenumbers is similar, at a time of $\mathcal{O}\left(t_{2}\right)$, and is periodic and bounded with a period $T=390.19 \mathrm{~s}$ which is of order $\mathcal{O}\left(t_{3}\right)$. 
According to Hasselmann's kinetic equation the time evolution of the wave action depends only on exact resonant waves. With the settings of cases $\mathbf{c}$ and $\mathbf{d}$ it is possible to have triads that are in exact resonance. The dashed line on the right panel of Figure 2 shows such triads. According to the GKE the time evolution of exact resonant triads blows up.

\section{Comparison between GKE and JKE-Bounded Solutions}

Having studied cases with $D<0$, it is instructive to compare the solutions of the GKE with those of the JKE for the cases $\mathbf{b}$ and $\mathbf{d}$ where both are periodic and bounded. These cases are depicted by squares $(p=0.2, \epsilon=0.01)$ and $(p=0.32, q=0.05)$ in Figure 2 .

Figure 5 shows the time evolution for case $\mathbf{b}$. The upper panel shows the solution of the GKE with period $T=192.51 \mathrm{~s}$, see (56), while the lower panel shows the solution of the JKE with a period of $T=198.07 \mathrm{~s}$. In both cases the period of the solution is $\mathcal{O}\left(t_{2}\right)$ and the qualitative behavior of both solutions is rather similar.

In Figure 5 one can see behavior similar to the stochastic Fermi-Pasta-Ulam recurrence (FPU recurrence), which is usually related to the long time behavior of the deterministic Benjamin-Feir instability. Details of the FPU recurrence can be found in Stiassnie and Kroszynski [15]. For stochastic recurrence derived from the Alber equation see Stiassnie, Regev and Agnon [16].

\section{Case $\mathbf{b}$}
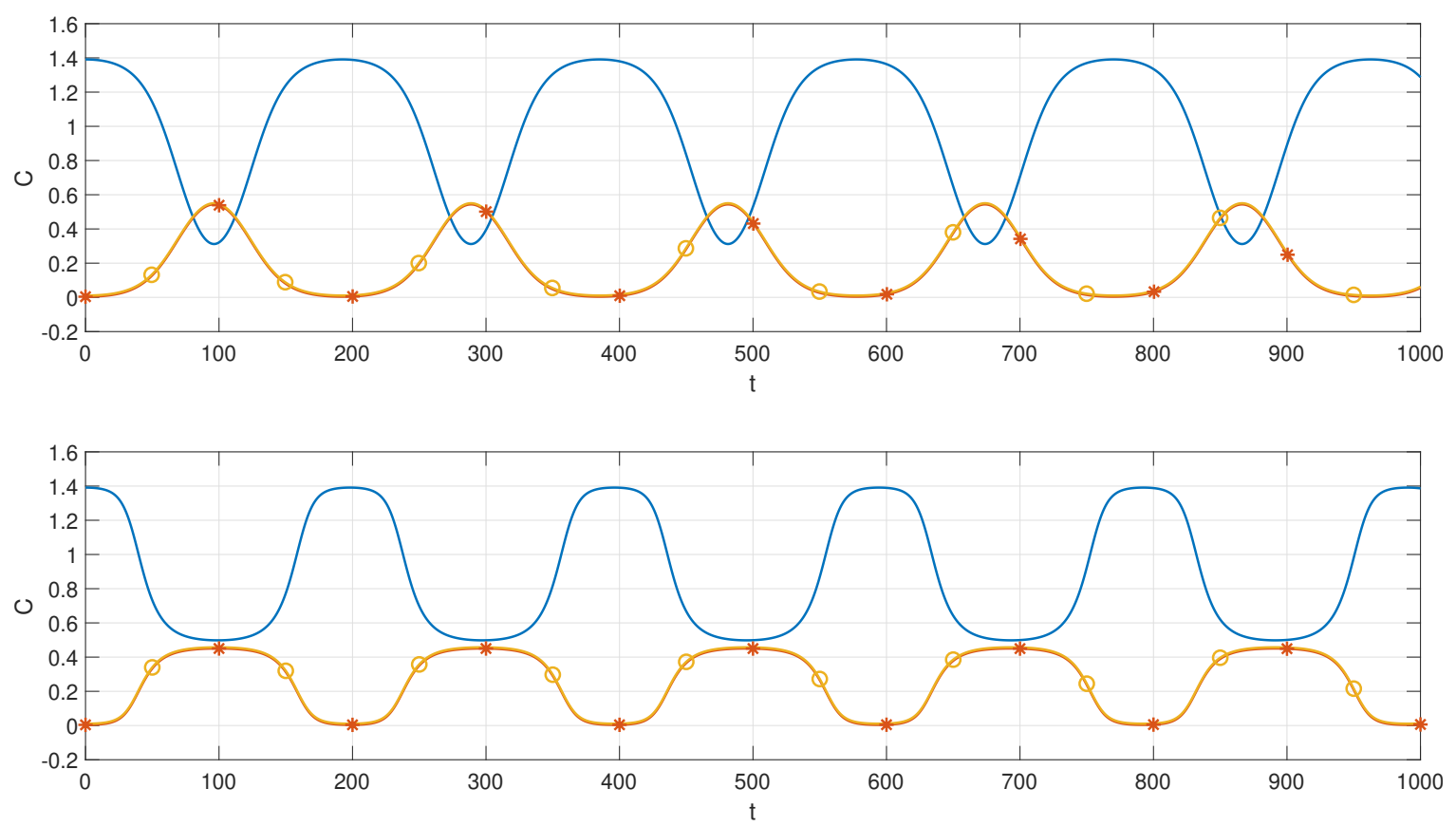

Figure 5. Time evolution of $C_{a}$ in blue (solid), $C_{b}$ in yellow (circles) and $C_{c}$ in red (asterisks) for ( $p=0.2, \epsilon=0.01)$. Upper panel: Solution of the GKE. The period is $T=192.51 \mathrm{~s}$. Lower panel: Solution of the JKE. The period is $T=198.07 \mathrm{~s}$. The time $t$ is in seconds.

Case d, depicted in Figure 6, shows considerable similarity between the solutions of the GKE and solutions of the JKE. The periods are $T=94.22 \mathrm{~s}$ for the GKE and $T=80.42 \mathrm{~s}$ for JKE, both of $\mathcal{O}\left(t_{2}\right)$. 


\section{Case d}
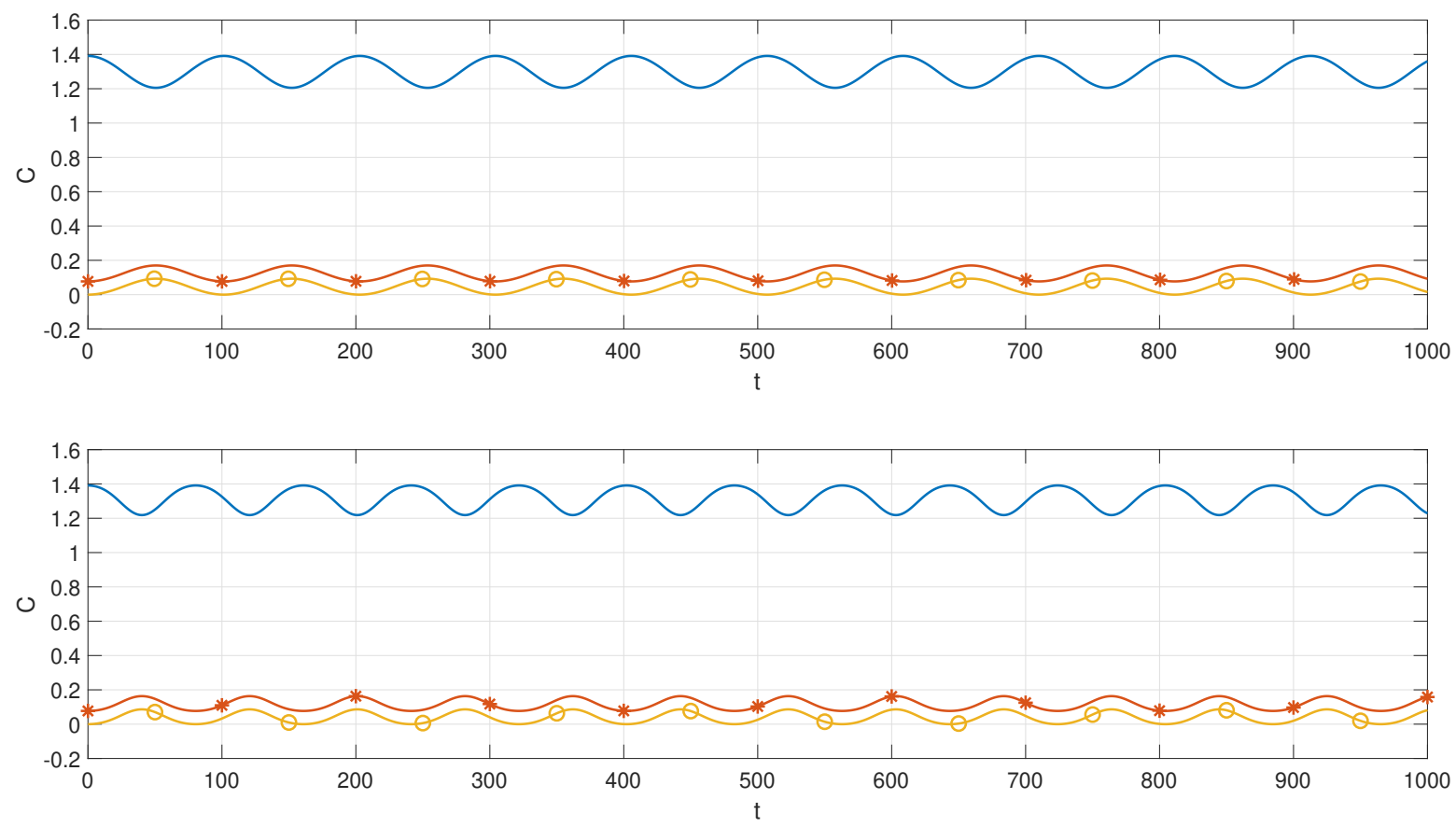

Figure 6. Time evolution of $C_{a}$ in blue (solid), $C_{b}$ in yellow (circles) and $C_{c}$ in red (asterisks) for $(p=0.32, q=0.05)$. Upper panel: Solution of the GKE. The period is $T=94.22 \mathrm{~s}$. Lower panel: Solution of the JKE. The period is $T=80.42 \mathrm{~s}$. The time $t$ is in seconds.

\section{Discussion and Concluding Remarks}

\subsection{Time Scales}

In Hasselmann's kinetic equation it is assumed that the wave field evolves on the slow time scale $\mathcal{O}\left(t_{4}\right)$, and only exactly resonant quartets are taken into account. In all of the examples presented here the degenerated quartet of waves is in near resonance, as indicated by the value of the detuning $\Delta_{a, a}^{b, c}$ in Table 1. According to the exactly resonant kinetic theory there should be no temporal evolution for such initial data, which in turn is consistent with the assumption that the wave field can be described entirely on the slow $t_{4}$ scale.

As pointed out by Janssen, the solutions of the JKE (15) capture changes that happen on the much faster time-scale $t_{2}$. Indeed, all of our simulations show an initial monotonic evolution on this $t_{2}$ scale. Nevertheless the period $T=2 \pi / \Delta$, with $\Delta$ given in Table 1 , is of $\mathcal{O}\left(t_{4}\right)$ for case $\mathbf{a}, \mathcal{O}\left(t_{3}\right)$ for case $\mathbf{c}$ and $\mathcal{O}\left(t_{2}\right)$ for cases $\mathbf{b}$ and $\mathbf{d}$.

\subsection{Assumption (i), Weak Non-Gaussianity}

Both the GKE and the JKE are derived using the ordering assumption, in Equation (7), that $C_{j}=\mathcal{O}\left(\varepsilon^{2}\right)$ and $\kappa=o\left(\varepsilon^{4}\right)$, where $\varepsilon$ is a typical wave steepness. In order to verify this assumption we compute the following ratio

$$
\delta=\frac{\max _{0 \leq t \leq 1000}\left|\kappa_{a, a, b, c}\right|}{C_{a}^{2}(0)}
$$

in all the examples presented here.

The results are summarized in Table 2. Note that $\varepsilon_{a}=0.15$ and that for all cases $\delta=\mathcal{O}\left(\varepsilon_{a}\right)$. Hence the computed results support the ordering of the cumulants in terms of $\varepsilon$. 
Table 2. Values of $\delta=\max _{0 \leq t \leq 1000}\left|\kappa_{a, a, b, c}(t)\right| / C_{a}^{2}(0)$ for computed solutions to the GKE and JKE in the four cases $\mathbf{a}-\mathbf{d}$.

\begin{tabular}{ccc}
\hline & GKE & JKE \\
\hline case a & blow up & 0.2516 \\
case b & 0.2268 & 0.2478 \\
case c & blow up & 0.2097 \\
case d & 0.1142 & 0.1460 \\
\hline
\end{tabular}

\subsection{Assumption (ii), Slow Temporal Evolution}

The key difference between the JKE and GKE is assumption (ii), that $C_{a} C_{b} C_{c}-C_{a}^{2}\left(C_{b}+C_{c}\right) / 2$ varies slowly compared with $\exp \left(i \Delta_{a, a}^{b, c} t\right)$. In fact, our numerical simulations with the JKE show that in cases a and $\mathbf{c}$ the period of $C_{a} C_{b} C_{c}-C_{a}^{2}\left(C_{b}+C_{c}\right) / 2$ is of the order of $\mathcal{O}\left(t_{4}\right)$ and $\mathcal{O}\left(t_{3}\right)$ respectively, although there are some rapid changes on the scale $\mathcal{O}\left(t_{2}\right)$.

In cases $\mathbf{b}$ and $\mathbf{d} C_{a} C_{b} C_{c}-C_{a}^{2}\left(C_{b}+C_{c}\right) / 2$ has variations on the $\mathcal{O}\left(t_{2}\right)$ scale, which is also the time scale of their period. Note that the solution of the JKE has the same period as the oscillatory term $e^{i \Delta_{a, a}^{b, c} t}$, see formula (71). This contradicts assumption (ii) for the JKE. Nevertheless, the surprising agreement between the GKE and the JKE in cases $\mathbf{b}$ and $\mathbf{d}$ suggests that the approximation may be valid, but it is not clear at this stage how it could be justified.

\subsection{Numerical Computations}

As mentioned in Section 5, Equation (71) was not used to compute solutions of the JKE because it is practically simpler to integrate the JKE numerically. However, for case c, (71) was used as a consistency check.

We used the numerical solution for $C_{a}, C_{b}$ and $C_{c}$ and through relations (27)-(29) obtained $z(t)$ as

$$
z(t)=\frac{C_{a}-2 C_{b}-2 C_{c}}{12 T_{a, a, b, c}\left(\rho_{a}-2 \rho_{b}-2 \rho_{c}\right)},
$$

which was used to compute both sides of (71). We subsequently measured the relative error at each time step, and found it always to be below $2.91 \times 10^{-6}$.

For the GKE the exact formulas (54) and (62) can be used directly to obtain the time evolution of the system. However, for completeness' sake, we also considered a direct numerical integration of (18)-(21) using MATLAB ode15s as before. For the bounded cases $\mathbf{b}$ and $\mathbf{d}$ we obtained a relative error between the exact and the numerical solutions below $2.8 \times 10^{-8}$ and $6.1 \times 10^{-10}$ at each time step from 0 to $1000 \mathrm{~s}$, respectively. For the unbounded cases a and $\mathbf{c}$ we measured the difference at each time step between 0 and $t_{f}=T_{\infty}-1 \mathrm{~s}$, which exhibited a relative error of $1.9 \times 10^{-8}$ for case a and $2.8 \times 10^{-9}$ for case $\mathbf{c}$.

We also ran numerical simulations with the GKE including the Stokes correction term of Gramstad and Stiassnie, see [8]. Note that the GKE with the Stokes corrections still blows-up, with a blow-up time similar to that shown in Figures 3 and 4.

\subsection{Conclusions}

At this stage blow-up in the GKE seems unavoidable, although it has hitherto not been reported in studies applying the GKE to wave-fields with many modes. For degenerate quartets and given initial data, the roots of the polynomial $P_{4}$ determine the nature of the solutions of the GKE, but there is no clear physical distinction, in terms of wave slope or wave number, that would enable one to distinguish bounded cases from those which blow-up. It seems further study is warranted before a clear successor to Hasselmann's kinetic equation, which incorporates near-resonant interactions, can be identified. 
Author Contributions: Conceptualization, D.A. and M.S.; methodology, D.A., M.S. and R.S.; investigation, D.A., R.S. and M.S.; writing-original draft preparation, D.A.; writing-review and editing, D.A., M.S., and R.S.; funding acquisition, M.S.

Funding: This research was supported by the Israel Science Foundation (Grant 261/17).

Conflicts of Interest: The authors declare no conflict of interest.

\section{Appendix A}

A possible mechanism to circumvent blow-up for solutions to the GKE lies in the phase-mixing approach developed by Gramstad and Stiassnie [8], and further employed by Gramstad and Babanin [9]. This essentially consists in finding piece-wise solutions of the GKE.

The time interval is divided into smaller subintervals of the form $t_{0}<t_{1}<\ldots<t_{f}$ and the solution is found by solving GKE on every interval with initial values $\rho_{j}=C_{j}\left(t_{i}\right)$ and $\kappa_{j, m, n, p}\left(t_{i}\right)=0$, effectively re-imposing the quasi-stationarity assumption (iii) at various points in the time evolution. The resulting piece-wise solution is continuous for $C_{j}$ and discontinuous for $\kappa$.

We use the phase-mixing to overcome blow-up as follows: we divide the time interval of our simulations into $0<t_{1}<t_{f}=1000 \mathrm{~s}$ where $t_{1}$ is the earliest time that one of the wave action terms becomes zero. Then we reset the value of $\kappa_{a, a, b, c}\left(t_{1}\right)$ to zero and solve the GKE using new initial conditions $C_{j}\left(t_{1}\right)$. This reinitialization of the cumulant can be justified for vanishing $C_{j}$. If $C_{j}=0$ at time $t_{1}$, the underlying random variable $B_{j}\left(t_{1}\right)$ must vanish as well, which implies that any higher order moment involving $B_{j}$ must also vanish. It then follows from the definition of $\kappa$, Equation (7), that $\kappa_{a, a, b, c}\left(t_{1}\right)=0$.

In cases a and conly one reinitialization is needed to obtain bounded solutions, as shown in Figures A1 and A2. Subsequent to the phase-mixing at $t_{1}$ the solutions are bounded and periodic with periods $T=502.3 \mathrm{~s}$ and $T=536.3 \mathrm{~s}$ respectively, both within the time scale $\mathcal{O}\left(t_{3}\right)$.

\section{Case a}
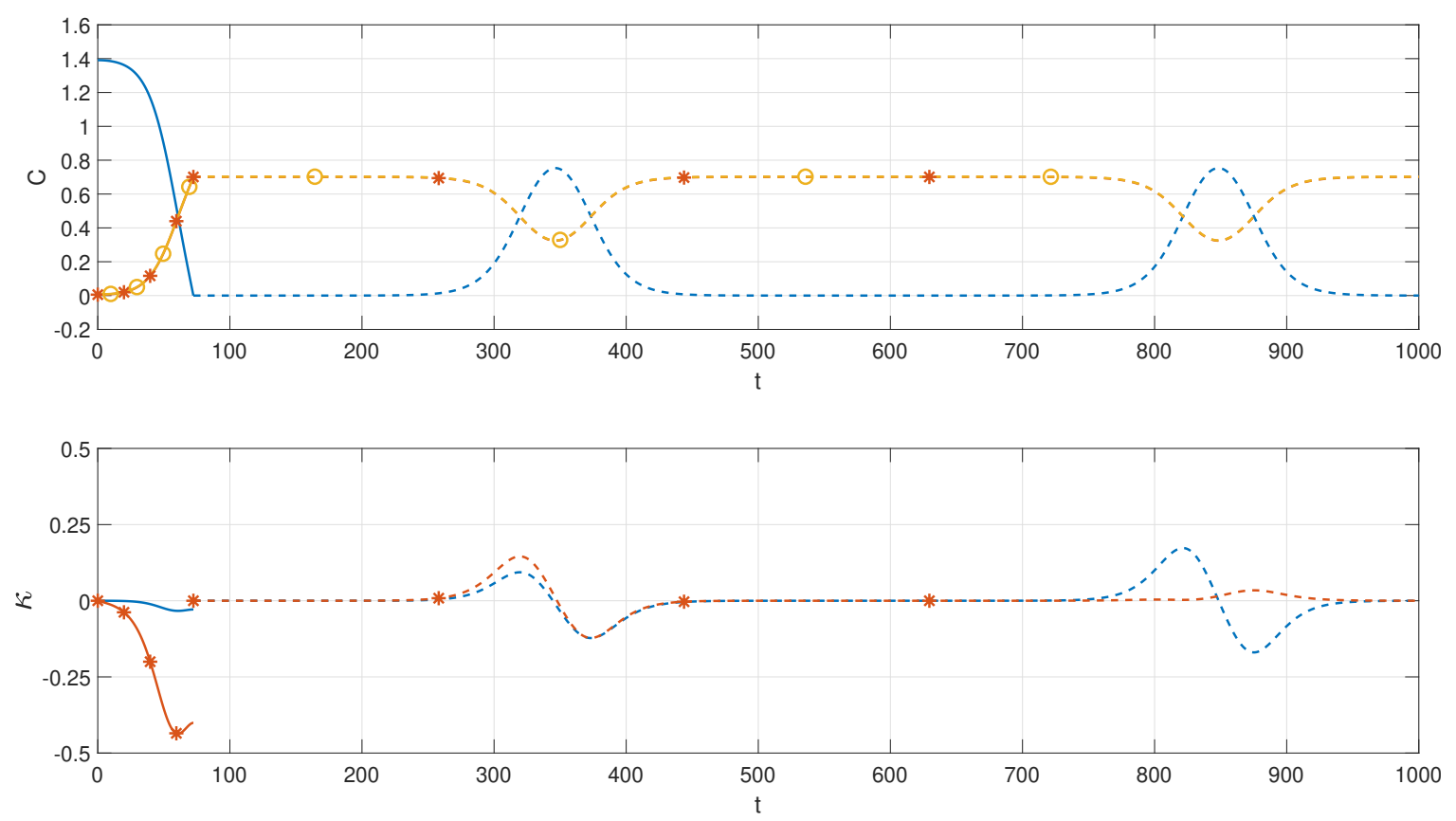

Figure A1. Upper panel: Solution of the GKE showing $C_{a}$ in blue, $C_{b}$ in yellow (circles) and $C_{c}$ in red (asterisks) for case a $(p=0.05, \epsilon=0.01)$, extended by phase-mixing at time $t_{1}=72.38 \mathrm{~s}$. Lower panel: Time evolution of $\kappa_{a, a, b, c}$. The real part of $\kappa_{a, a, b, c}$ is shown in blue and the imaginary part of $\kappa_{a, a, b, c}$ in red (asterisks). In all cases, solid lines denote the solution before phase-mixing, dashed lines subsequent to phase mixing. The time $t$ is in seconds. 


\section{Case c}
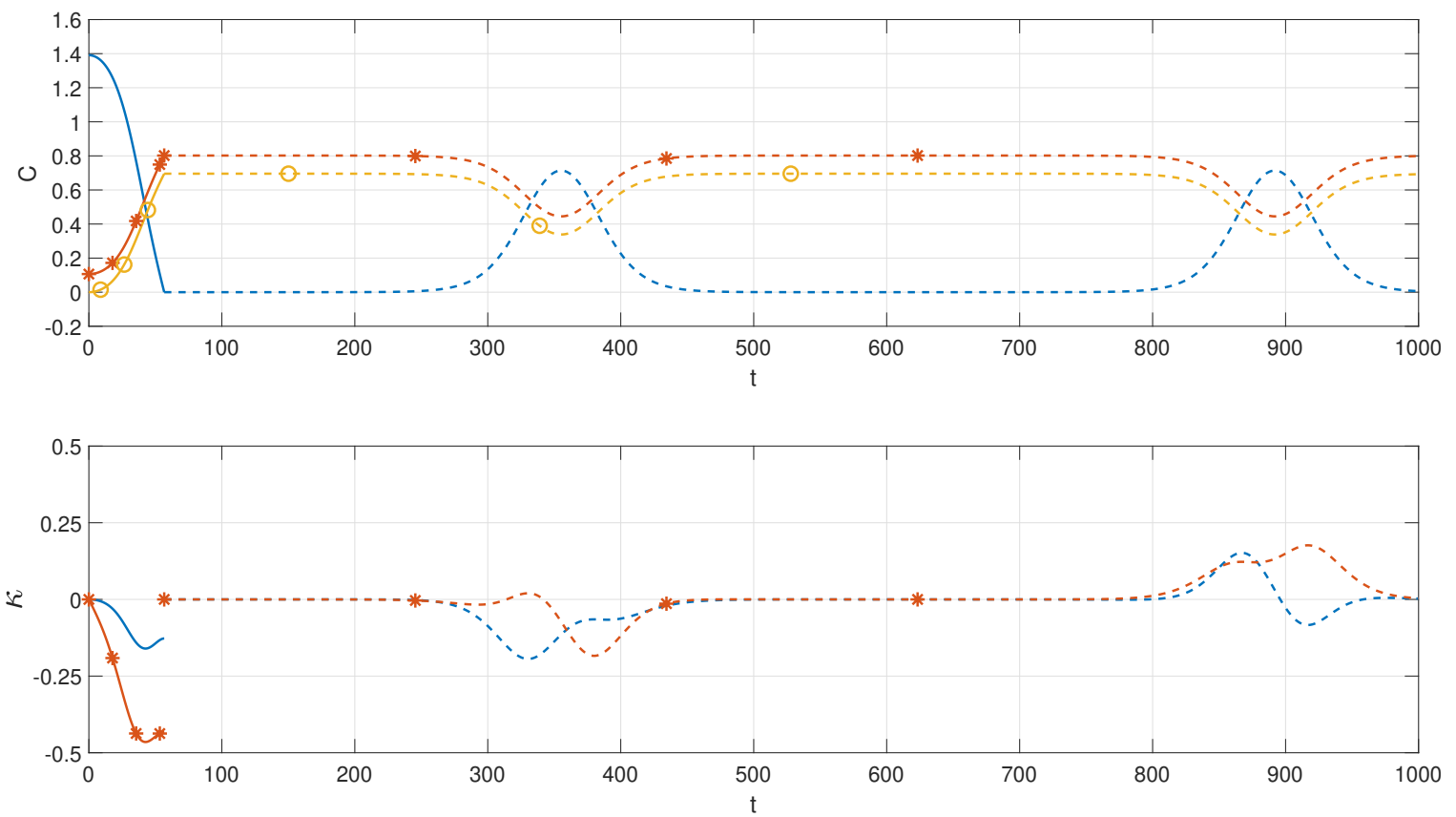

Figure A2. Upper panel: Solution of the GKE showing $C_{a}$ in blue, $C_{b}$ in yellow (circles) and $C_{c}$ in red (asterisks) for case $\mathbf{c}(p=0.16, q=0.05)$, extended by phase-mixing at time $t_{1}=56.66 \mathrm{~s}$. Lower panel: Time evolution of $\kappa_{a, a, b, c}$. The real part of $\kappa_{a, a, b, c}$ is shown in blue and the imaginary part of $\kappa_{a, a, b, c}$ in red (asterisks). In all cases, solid lines denote the solution before phase-mixing, dashed lines subsequent to phase mixing. The time $t$ is in seconds.

\section{References}

1. Phillips, O.M. On the dynamics of unsteady gravity waves of finite amplitude Part 1 . The elementary interactions. J. Fluid Mech. 1960, 9, 193. [CrossRef]

2. Zakharov, V.E. Stability of periodic waves of finite amplitude on the surface of a deep fluid. J. Appl. Mech. Tech. Phys. 1968, 9, 190-194. [CrossRef]

3. Krasitskii, V.P. On reduced equations in the Hamiltonian theory of weakly nonlinear surface waves. J. Fluid Mech. 1994, 272, 1-20. [CrossRef]

4. Hasselmann, K. On the non-linear energy transfer in a gravity-wave spectrum Part 1. General theory. J. Fluid Mech. 1962, 12, 481. [CrossRef]

5. Stiassnie, M.; Shemer, L. On the interaction of four water-waves. Wave Motion 2004, 41, 307-328. [CrossRef]

6. Janssen, P.A.E.M. Nonlinear Four-Wave Interactions and Freak Waves. J. Phys. Oceanogr. 2003, 33, 863-884. [CrossRef]

7. Annenkov, S.Y.; Shrira, V.I. Role of non-resonant interactions in the evolution of nonlinear random water wave fields. J. Fluid Mech. 2006, 561, 181. [CrossRef]

8. Gramstad, O.; Stiassnie, M. Phase-averaged equation for water waves. J. Fluid Mech. 2013, 718, $280-303$. [CrossRef]

9. Gramstad, O.; Babanin, A. The generalized kinetic equation as a model for the nonlinear transfer in third-generation wave models. Ocean Dyn. 2016, 66, 509-526. [CrossRef]

10. Stuhlmeier, R.; Stiassnie, M. Evolution of statistically inhomogeneous degenerate water wave quartets. Philos. Trans. R. Soc. A Math. Phys. Eng. Sci. 2019, 376, 20170101. [CrossRef] [PubMed]

11. Crawford, D.R.; Saffman, P.G.; Yuen, H.C. Evolution of a random inhomogeneous field of nonlinear deep-water gravity waves. Wave Motion 1980, 2, 1-16. [CrossRef]

12. Lvov, Y.V.; Binder, R.; Newell, A.C. Quantum weak turbulence with applications to semiconductor lasers. Phys. D Nonlinear Phenom. 1998, 121, 317-343. [CrossRef]

13. Irving, R.S. Integers, Polynomials, and Rings. Undergraduate Texts in Mathematics; Springer: New York, NY, USA, 2004. 
14. Byrd, P.F.; Friedman, M.D. Handbook of Elliptic Integrals for Engineers and Scientists; Springer: Berlin/Heidelberg, Germany, 1971. [CrossRef]

15. Stiassnie, M.; Kroszynski, U.I. Long-time evolution of an unstable water-wave train. J. Fluid Mech. 1982, 116, 207-225. [CrossRef]

16. Stiassnie, M.; Regev, A.; Agnon, Y. Recurrent solutions of Alber's equation for random water-wave fields. J. Fluid Mech. 2008, 598. [CrossRef]

(C) 2018 by the authors. Licensee MDPI, Basel, Switzerland. This article is an open access article distributed under the terms and conditions of the Creative Commons Attribution (CC BY) license (http://creativecommons.org/licenses/by/4.0/). 